\title{
Can lung ultrasound assist in the decision of weaning mechanically ventilated neonates?
}

\author{
Mohamed Adel Eltomey ${ }^{1 *}$, Ahmed Hany Shehata ${ }^{2}$, Mohamed Abd Ellatiff Nassar ${ }^{2}$ and \\ Abd Elrahman Mohamed Elmashad ${ }^{2}$
}

\begin{abstract}
Background: Mechanical ventilation is widely used in neonates presenting with respiratory distress; however, it is not without complications. Early weaning of neonates is vital however the process of extubation is difficult to determine precisely. The use of lung ultrasound in neonates is recently accepted as a reliable tool in the assessment of several lung conditions. The aim of this study was to assess the use of lung ultrasound score as a quantitative method to assist in the decision of weaning mechanically ventilated neonates.

Results: The study was conducted on 40 neonates admitted to the NICU with different causes of respiratory distress needing respiratory support by mechanical ventilation. Lung ultrasound was performed using a linear superficial probe at least three times, at admission, before switching mechanical ventilation mode and before weaning. The initial lung ultrasound score for all patients was between 9 and 36 (mean $25 \pm 6.97$, median 26) with the 11 patients initiated on Sync. Intermittent ventilation (SIMV) showing significantly lower scores than those initiated on assist/control ventilation (ACV). Patients successfully switched from ACV to SIMV showed significantly lower scores than those who failed. Patients successfully weaned from SIMV showed significantly lower scores than those who failed. ROC analysis showed a cut-off score of $\leq 14$ had $85 \%$ sensitivity and 100\% specificity for a successful switch between ACV to SIMV. It also showed that a score $\leq 6$ had $87.5 \%$ sensitivity and $100 \%$ specificity for successful extubation.
\end{abstract}

Conclusion: The use of quantitative lung ultrasound scores in assessment of mechanically ventilated neonates shows a great potential in aiding the process of weaning.

Keywords: Lung, Ultrasound, Mechanical ventilation, Neonates, Weaning

\section{Background}

Mechanical ventilation is widely used in the management of neonates especially preterm patients, even with recent advances in non-invasive respiratory support $[1,2]$. Despite its support for the respiratory system during its recovery from failure, it is not without complications and risks, including mortality and impairment of the nervous system development. So, when it comes for neonates and infants

\footnotetext{
*Correspondence: mohamed.eltomey@med.tanta.edu.eg

${ }^{1}$ Radiology and Medical Imaging Department, Faculty of Medicine, Tanta

University, Tanta, Egypt

Full list of author information is available at the end of the article
}

care, focus on early removal of mechanical ventilation should be considered to avoid such complications [3].

Failure of extubation is frequently seen in premature infants. The readiness of the patient for extubation is difficult to determine precisely and is usually based on the clinical judgment of the physician in charge of the case [2].

Lung ultrasound (LUS), which many radiologists thought was unfeasible, has gained popularity in the intensive care units being used as a rapid aid for decision making in critically ill patients. It has the advantages of being rapid, portable, and lacking the hazard of ionizing radiation of radiography and computed tomography [4]. Lack of ionizing radiation makes it a suitable tool for imaging neonates, 


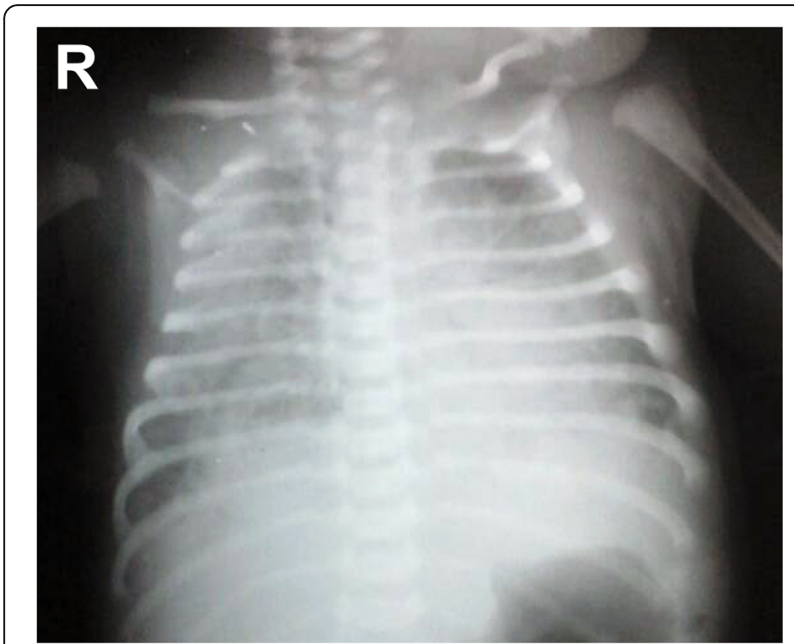

Fig. 1 Chest radiograph of a 1-day-old neonate showing a bilateral decrease of the lung volumes with diffuse granular opacities conforming with the picture of surfactant deficiency disease

as several studies showed its accuracy in the diagnosis of several neonatal lung conditions including, but not limited to, respiratory distress syndrome and neonatal pneumonia [5-9]. Findings of lung ultrasound are based on the fact that its waves are not transmitted through a normally aerated lung and that only pleural lines can be seen. The reverberation artifact seen in normal aerated lungs is known as A-lines. Loss of lung aeration as in cases of pulmonary edema, for example, will change the appearance of the artifact caused by lung into vertical lines known as B-lines $[4,10]$.
The aim of this study was to assess the utility of lung ultrasound score, as a quantitative method, to assist in the decision of weaning mechanically ventilated neonates.

\section{Methods}

The study was a prospective observational study including 40 neonates suffering from respiratory distress of different etiologies and requiring mechanical ventilation regardless of their gestation age at the time of admission. Patients with proven congenital heart disease, chest deformities, multiple complex congenital anomalies, neurological affection (i.e., Hypoxic-ischemic encephalopathy or intracranial hemorrhage) and congenital infections were excluded from the study. The study was conducted in the NICU unit at our institution, starting in June 2016 and ending December 2016. Ethical committee approval of our institution was obtained and informed consent was taken from the parents or guardians of the patients.

Patients were subjected to history taking (prenatal, natal and post-natal), general and local examination of the chest, heart, and abdomen as well a neurological examination. Laboratory investigations included complete blood picture, serum C-reactive protein, serum electrolytes, and capillary blood gases.

Imaging investigations included plain radiography of the chest at admission and daily for follow-up. Radiographs were performed in the supine anteroposterior position using a mobile X-ray machine and computed radiography plates.

Lung ultrasound was performed using the multifrequency superficial linear probe $(6-12 \mathrm{MHz})$ installed on

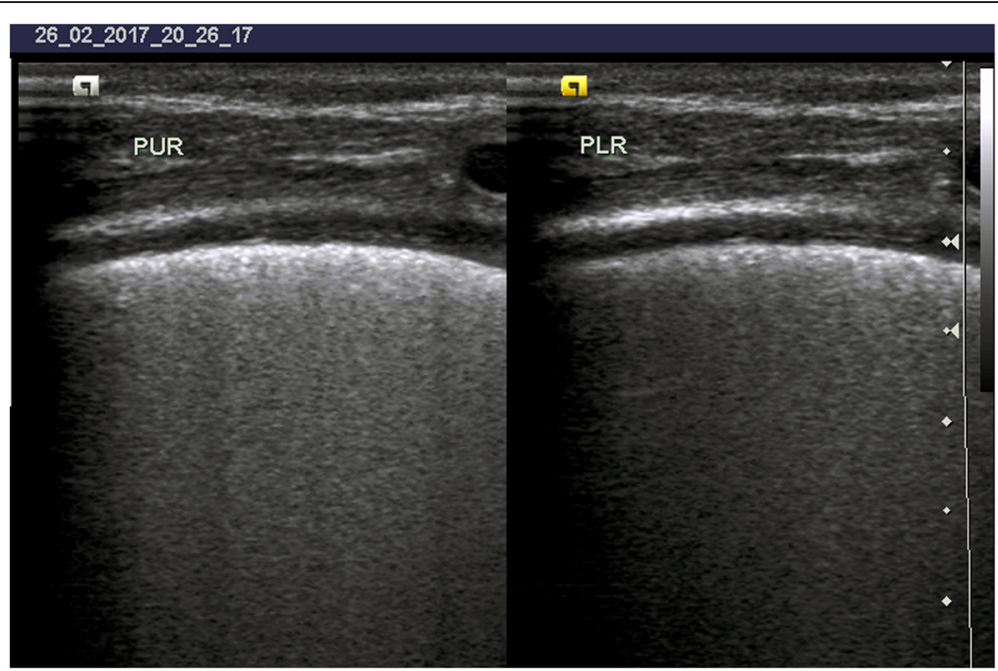

Fig. 2 B-mode ultrasound of the same patient on the day of admission to the NICU. Scanning of the posterior upper and lower zones of the right lung showed of a total loss of A-lines with coalescent B-lines causing curtain appearance giving a local score of 2. The total lung ultrasound score of the patient was 28 
Table 1 Showing their initial lung ultrasound score for neonates at the time of admission

\begin{tabular}{llll}
\hline & Initial LUS (all 40 neonates) & LUS ACV (no. 29) & LUS SIMV (no. 11) \\
\hline Range & $9-36$ & $9-36$ & $9-32$ \\
Mean \pm SD & $25 \pm 6.97$ & $27 \pm 5.87$ & $19 \pm 6.28$ \\
Median & 26 & 28 & 19 \\
\hline
\end{tabular}

the Siemens Acuson X300 ultrasound machine (Siemens Health Care GmbH, Erlangen, Germany). Lung ultrasound was performed at least three times, at admission, before changing ventilation mode and before extubation. Additional scans were required in patients showing deterioration after changing the ventilation mode for follow-up and re-assessment. The lung ultrasound score proposed by Bouhemad et al. [10] was used to standardize the examination for all patients. Each hemithorax was divided into 6 regions, summing 12 zones for both sides. Upper and lower halves which were further divided into anterior, medial, and lateral zones. The anterior zone is between the medial aspect of the sternum and the anterior axillary line, the medial zone between anterior and posterior axillary lines, and the posterior zone posterior to the posterior axillary line. The probe was applied along the intercostal spaces in each zone, and the degree of aeration was assessed and given a score according to the findings. Normal aeration showing A-lines and lung sliding was given a score of 0,3 , or more separated B-lines denoting mild aeration loss were given a score of 1 , marked aeration loss showing coalescent B-lines or curtain sign was given a score of 2 and lastly if lung consolidation was present it was given a score of 3 . The maximum score for both lungs was 36 . Ultrasound examinations were performed by a radiologist with an experience of more than 15 years in the field of pediatric imaging and ultrasound. In a case of emergency, examinations were performed by the senior radiology resident on call, trained by the radiologist. Images of the lung zones were stored on the machine and later revised by a radiologist. The entire examination took less than $10 \mathrm{~min}$ to perform. The decision of weaning patients in this study was not dependent solely on the lung ultrasound scores but on several clinical, biochemical, and radiological parameters.

Patients were mechanically ventilated using assist/control (AC) ventilation in 29 patients and sync intermittent mechanical ventilation (SIMV) in 11 patients. The 29 patients on $\mathrm{AC}$ ventilation mode were then switched to SIMV before weaning and extubation. Patients were followed up clinically for $48 \mathrm{~h}$ after extubation for assessment of its success or failure and the need for reintubation and mechanical ventilation.

Statistical analysis included descriptive statistics for age, weight, and frequency of variables. Analytic statistics included the Mann-Whitney $U$ test for comparison between the groups and ROC curve analysis. Statistics were performed using Medcalc 18 statistical software (MedCalc Software bvba).

\section{Results}

The study included 40 neonates, 24 males and 16 females. The gestational age at delivery was 28 to 40 weeks

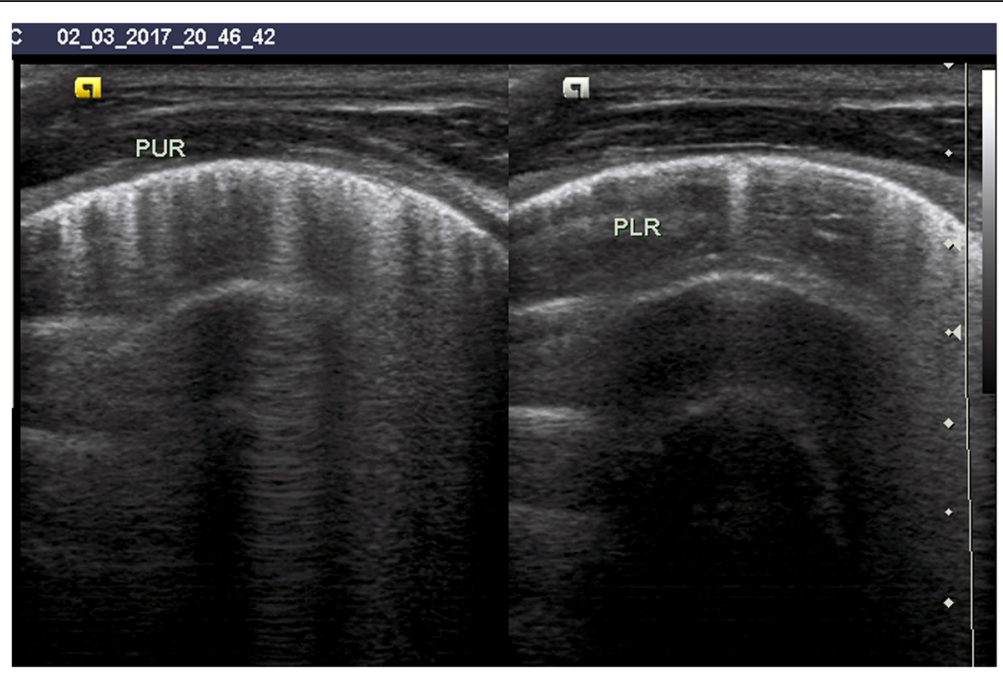

Fig. 3 B-mode ultrasound in the same patient after 3 days of treatment and being on assisted controlled (AC) ventilation. Scanning of the posterior upper and lower zones of the right lung showed improvement of lung aeration with multiple separated B-lines giving a local score of 1. The total lung ultrasound score of the patient was 12 
Table 2 Showing LUS scores for neonates on AC ventilation before switching to SIMV and the difference in scores between patients with successful and failed switch

\begin{tabular}{llll}
\hline & LUS ACV (all 29 neonates before switch) & LUS ACV Successful switch (20 neonates) & LUS ACV failed switch (9 neonates) \\
\hline Range & $9-19$ & $9-15$ & $15-19$ \\
Mean \pm SD & $14 \pm 2.64$ & $13 \pm 1.78$ & $17 \pm 1.13$ \\
Median & 14 & 13 & 17 \\
\hline
\end{tabular}

with a mean age of $35 \pm 3.7$ weeks. The birth weight varied between 1.3 to $3.9 \mathrm{~kg}$ with a mean weight of $2.68 \pm$ $0.76 \mathrm{~kg}$. The mode of delivery was Caesarian section in 29 patients and normal delivery in the remaining 11 patients.

The final diagnosis of the patients was surfactant deficiency disease in 21 patients (Fig. 1), transient tachypnea of newborn in 15 patients, meconium aspiration in 3 patients, and 1 patient with neonatal pneumonia.

The initial lung ultrasound score for the 40 neonates at admission was between 9 and 36 (mean $25 \pm 6.97$, median 26) (Fig. 2). Assist/control ventilation (ACV) was used in 29 patients and in the other 11 patients sync intermittent ventilation (SIMV) was used. The initial LUS for the 29 patients on ACV was between 9 and 36 (mean $27 \pm 5.87$, median 28) and for the 11 patients on SIMV was between 9 and 32 (mean $19 \pm 6.28$, median 19). Patients ventilated on SIMV showed a statistically significant lower LUS score when compared to those on ACV using the MannWhitney $U$ test with a $p<0.002$ (Table 1 ).

The lung ultrasound score for the 29 patients on ACV mode before switching to SIMV was between 9 and 19 (mean 14 \pm 2.64 , median 14). After switching to SIMV mode, 9 patients showed deterioration and needed to be switched back to ACV mode, and the LUS scores for these patients were between 15 and 19 (mean $17 \pm 1.13$, median 17). The 20 patients showing successful switch LUS score was between 9 and 15 (mean $13 \pm 1.78$, median 13) (Fig. 3). Patients showing a successful switch showed statistically a significant lower LUS score with a $p<0.001$ using the Mann-Whitney $U$ test (Table 2). The 9 patients showing failed switch of the ventilation mode were further followed up until their LUS decreased and eventually all were switched to SIMV before weaning, their LUS was between 10 and 15 (mean $12 \pm 1.66$, median 12), their scores were significantly lower than their score at the initial switch attempt with $p=0.0005$.

ROC curve analysis of the LUS for the 29 patients LUS at the initial switch attempt showed that a LUS score cut-off value of $\leq 14$ had $85 \%$ sensitivity and $100 \%$ specificity for a successful switch between ACV to SIMV (Fig. 4).

The LUS for all 40 neonates on SIMV before weaning and extubation was between 0 and 13 (mean $5 \pm 3.03$, median 5). After $48 \mathrm{~h}, 8$ patients needed reintubation and ventilation their LUS was between 7 and 13 (mean
$9 \pm 1.92$, median 9). The LUS score for the 32 patients with successful extubation was between 0 and 9 (mean $4 \pm 2.42$, median 5) (Fig. 5). Statistical analysis using Mann-Whitney $U$ test showed a significant difference between the LUS score of patients with successful and those with failed extubation with $p$ value 0.0001 (Table 3). ROC analysis of the LUS before extubation showed that a score $\leq 6$ had $87.5 \%$ sensitivity and $100 \%$ specificity for successful extubation (Fig. 6).

\section{Discussion}

The process of weaning and extubation from mechanical ventilation is still a challenge and remains an inexact science [2].

In this study, our main objective was to explore the potential of bedside lung ultrasound in the neonatal intensive care unit as a decision-making aid in the process of weaning neonates from mechanical ventilation. In this study, we used the lung ultrasound score as proposed by Bouhemad et al. [10] as a quantitative method of assessing the degree of lung aeration. The initial LUS of the patients, in the current study, at admission was between

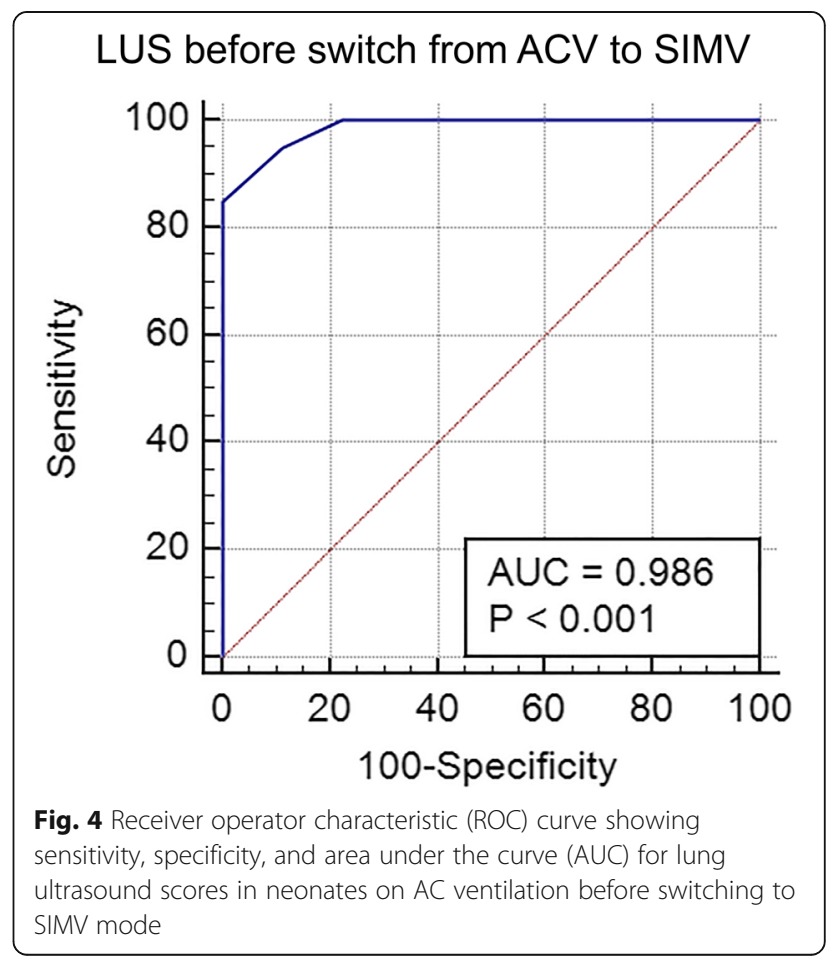




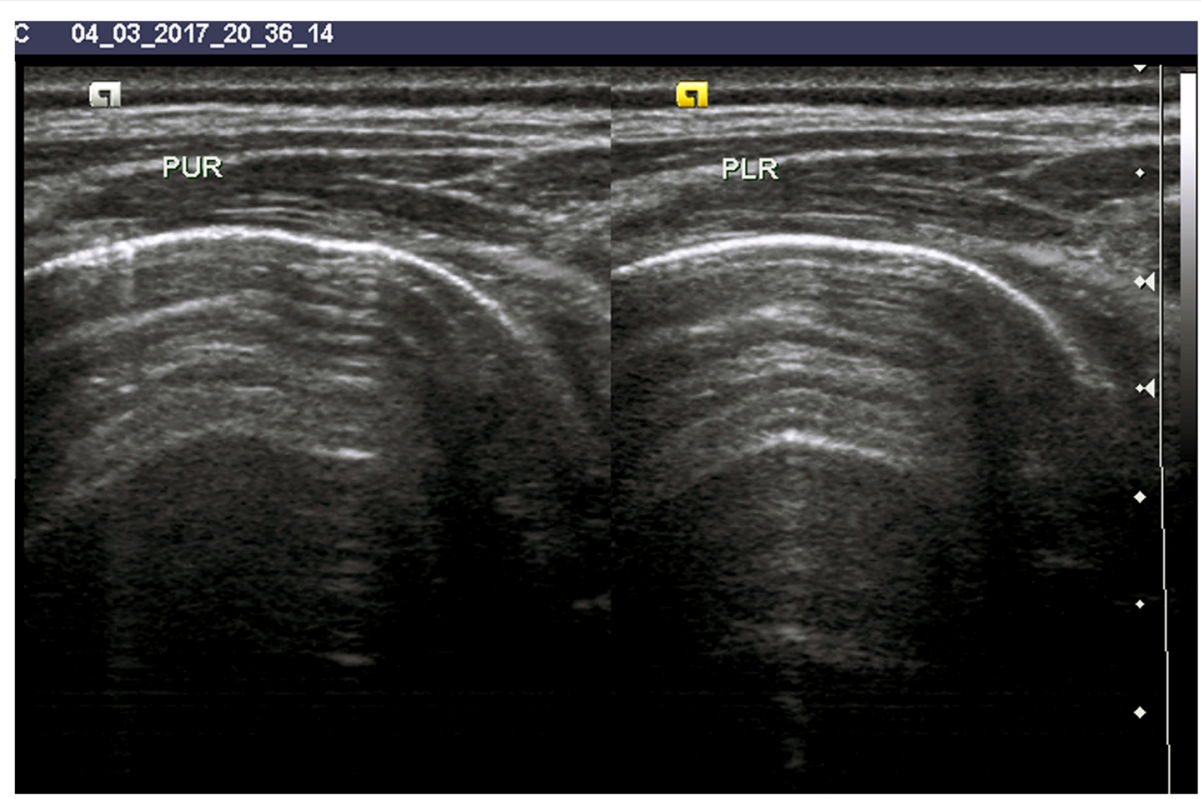

Fig. 5 B-mode ultrasound in the same patient before extubation and after switching to synchronized intermittent mechanical ventilation SIMV. Scanning of the posterior upper and lower zones of the right lung showed improvement of lung aeration with regain of normal A-lines giving a local score of 0 . The total lung ultrasound score of the patient was 3, and the patient was successfully extubated

9 and 36, and it was found that patients initially ventilated on SIMV mode showed significantly lower scores (mean $19 \pm 6.28$, median 19) than those starting on AC mode (mean $27 \pm 5.87$, median 28). Another finding was that neonates showing a successful switch from AC mode to SIMV showed significantly lower LUS. Hummler et al. [11] and Kapasi et al. [12] stated that several modes of mechanical ventilation are present, including assist/control (AC) and synchronized intermittent mechanical ventilation (SIMV). The choice of which mode to use, between the latter two, is still a matter of debate, as the available data does not clearly show the superiority of one mode over the other $[11,12]$. In our institution, neonatologists prefer using SIMV mode of ventilation before weaning the patients, which may explain the coincidence of patients initially ventilated on SIMV mode having lower scores than those initiated on AC mode. It may also explain the success of switching patients from AC mode to SIMV mode due to their lower scores reflecting better clinical and laboratory findings. It is noteworthy also to mention that findings in this study are also concordant with those of Raimondi et al. [13] who showed that lung ultrasound can predict the need of respiratory support in neonates; however, they used a proposed lung ultrasound pattern grade which they described as semiquantitative, not the quantitative lung ultrasound score used in this study [13]. Based on their proposed grading system, grade 1 in their study would correlate to a score around 24 in our study, if a bilateral symmetrical involvement of both lungs was present.

In this study, patients who showed successful switching from AC to SIMV had lower lung ultrasound scores (mean $13 \pm 1.78$, median 13) when compared to those who showed failed switch and distress (mean $17 \pm 1.1 .13$, median 17), a cut-off value of $\leq 14$ showed $85 \%$ sensitivity and $100 \%$ specificity for a successful switch between ACV to SIMV. It was also found the LUS score in patients with successful extubation (mean $4 \pm 2.42$, median 5 ) at the end of the study were significantly lower than those showing extubation failure (mean $9 \pm 1.92$, median

Table 3 Showing LUS scores for neonates on SIMV ventilation before weaning and extubation and the difference in scores between patients with successful and failed extubation

\begin{tabular}{llll}
\hline & LUS SIMV (all 40 neonates before extubation) & LUS SIMV successful extubation (32 neonates) & LUS SIMV failed extubation (8 neonates) \\
\hline Range & $0-13$ & $0-9$ & $7-13$ \\
Mean \pm SD & $5 \pm 3.03$ & $4 \pm 2.42$ & $9 \pm 1.92$ \\
Median & 5 & 5 & 9 \\
\hline
\end{tabular}




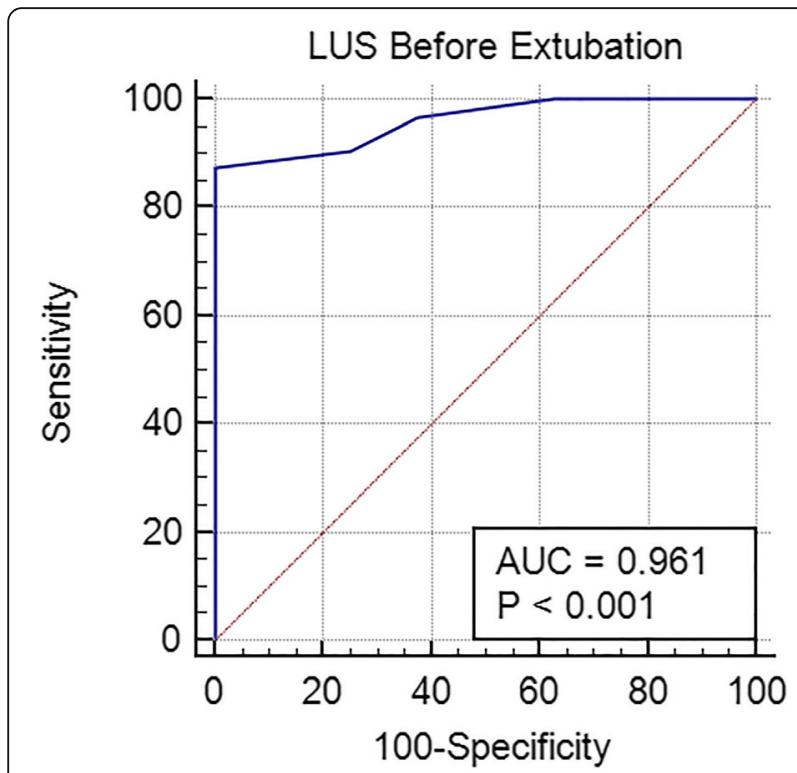

Fig. 6 Receiver operator characteristic (ROC) curve showing sensitivity, specificity, and area under the curve (AUC) for lung ultrasound scores in neonates on SIMV mode before extubation

9) and the need for re-ventilation. A cut-off score of $\leq 6$ was found to have a $87.5 \%$ sensitivity and $100 \%$ specificity for successful extubation.

Soummer et al. [14] in their research on adults showed that patients with higher lung ultrasound scores are prone to post-extubation distress, and their proposed cut-off value was $\geq 17$. They also proposed a cut-off value of $<13$ as a good indicator of extubation success [14]. Soliman et al. [15] proposed a cut-off value of $\geq$ 15.5 as an indicator of extubation failure [15]. The relative differences in their results and cut-off values with our study could be probably explained by the difference in the studies population ages and methods of mechanical ventilation used; however, the general common finding is that patients with lower lung ultrasound scores show a better chance of extubation success.

Raimondi et al. [16] showed in their study that lung ultrasound is a useful tool in predicting non-invasive ventilation failure in neonates, using the previously mentioned ultrasound pattern grade not the lung ultrasound score [16]. In their study patients with grade I pattern (white lung) needed intubation and mechanical ventilation while patients with grades 2 and 3 were managed by non-invasive ventilation or conservatively. Concordance of results between their study and ours is present despite the different approaches to lung affection grading system.

Studies using quantitative lung ultrasound score as an indicator of lung aeration and the need of surfactant administration in cases of respiratory distress in newborns are present $[17,18]$; however, we could not find a study discussing the use of this score in relation to mechanical ventilation in the same age group.

\section{Conclusion}

In view of our findings, we can conclude that the use of quantitative lung ultrasound score shows great potential as a reliable tool in the assessment of mechanically ventilated neonates and aid in the decision-making during the process of weaning as in adults, despite the relatively small number of patients this study was conducted on.

\section{Abbreviations}

ACV: Assist/control ventilation; LUS: Lung ultrasound; ROC: Receiver operator characteristics; SIMV: Synchronized (Sync.) Intermittent mechanical ventilation

\section{Acknowledgements}

Not applicable.

\section{Authors' contributions}

All authors have appraised the article and actively contributed to the work. ME contributed to the imaging of patients, manuscript writing, statistical analysis, and revision of the manuscript. AS contributed to the scanning of the patients and collection of data. MN helped in the revision of clinical findings and manuscript revision. AH contributed to the idea of the research, facilitation of data collection, and overall revision of the manuscript. All authors read and approved the final manuscript.

\section{Funding}

The authors declare that the study was self-funded and no funding was obtained from the manufacturers of the machines used, from any of its representatives, or from any other organization.

\section{Availability of data and materials}

The data sets generated and/or analyzed in this study are available through the corresponding author on reasonable request.

\section{Ethics approval and consent to participate}

This study was approved by the Research Ethics Committee of the Faculty of Medicine at Tanta University in Egypt in April 2016; reference number of approval: 30897/04/16. All parents or guardians of the patients included in this study gave written informed consent to participate in this research.

\section{Consent for publication}

All parents or guardians of the patients included in this research gave written informed consent to publish the data contained within this study. The authors grant the publisher the consent for publication of this work.

\section{Competing interests}

The authors declare that they have no competing interests.

\section{Author details}

${ }^{1}$ Radiology and Medical Imaging Department, Faculty of Medicine, Tanta University, Tanta, Egypt. ${ }^{2}$ Pediatrics Department, Faculty of Medicine, Tanta University, Tanta, Egypt.

Received: 17 August 2019 Accepted: 23 September 2019

Published online: 12 November 2019

\section{References}

1. Walsh MC, Morris BH, Wrage LA, Vohr BR, Poole WK, Tyson JE et al (2005) Extremely low birthweight neonates with protracted ventilation: mortality and 18-month neurodevelopmental outcomes. J Pediatr 146(6):798-804

2. Sant'Anna GM, Keszler M (2012) Weaning infants from mechanical ventilation. Clin Perinatol 39(3):543-562

3. Kamlin COF, Davis PG, Morley CJ (2006) Predicting successful extubation of very low birthweight infants. Arch Dis Child Fetal Neonatal Ed 91(3): F180-F1F3 
4. Lichtenstein DA (2014) Lung ultrasound in the critically ill. Ann Intensive Care 4(1):1

5. Seif El Dien HM, Abd ElLatif DAK (2013) The value of bedside lung ultrasonography in diagnosis of neonatal pneumonia. Egypt Journal of Radiology and Nuclear Medicine 44(2):339-347

6. Liu JMP, Cao H-YM, Wang H-WM, Kong X-YMP (2014) The role of lung ultrasound in diagnosis of respiratory distress syndrome in newborn infants. Iran J Pediatr 24(2):147-154

7. Sawires HK, Abdel Ghany EA, Hussein NF, Seif HM (2015) Use of lung ultrasound in detection of complications of respiratory distress syndrome. Ultrasound Med Biol 41(9):2319-2325

8. Lichtenstein DA, Mauriat P (2012) Lung ultrasound in the critically ill neonate. Curr Pediatr Rev 8(3):217-223

9. Raimondi F, Yousef N, Migliaro F, Capasso L, De Luca D (2108) Point-of-care lung ultrasound in neonatology: classification into descriptive and functional applications. Pediatr Res

10. Bouhemad B, Mongodi S, Via G, Rouquette I (2015) Ultrasound for "lung monitoring" of ventilated patients. Anesthesiology 122(2):437-447

11. Hummler H, Gerhardt T, Gonzalez A, Claure N, Everett R, Bancalari E (1996) Influence of different methods of synchronized mechanical ventilation on ventilation, gas exchange, patient effort, and blood pressure fluctuations in premature neonates. Pediatr Pulmonol 22(5):305-313

12. Kapasi M, Fujino Y, Kirmse M, Catlin EA, Kacmarek RM (2001) Effort and work of breathing in neonates during assisted patient-triggered ventilation. Pediatr Crit Care Med 2(1):9-16

13. Raimondi F, Migliaro F, Sodano A, Umbaldo A, Romano A, Vallone G et al (2012) Can neonatal lung ultrasound monitor fluid clearance and predict the need of respiratory support? Crit Care 16(6):R220

14. Soummer A, Perbet S, Brisson H, Arbelot C, Constantin JM, Lu Q et al (2012) Ultrasound assessment of lung aeration loss during a successful weaning trial predicts postextubation distress*. Crit Care Med 40(7):2064-2072

15. Soliman SB, Ragab F, Soliman RA, Gaber A, Kamal A (2019) Chest ultrasound in predication of weaning failure. Open Access Maced J Med Sci 7(7):1143-1147

16. Raimondi F, Migliaro F, Sodano A, Ferrara T, Lama S, Vallone G et al (2014) Use of neonatal chest ultrasound to predict noninvasive ventilation failure. Pediatrics. 134(4):e1089-ele94

17. De Martino L, Yousef N, Ben-Ammar R, Raimondi F, Shankar-Aguilera S, De Luca D (2018) Lung ultrasound score predicts surfactant need in extremely preterm neonates. Pediatrics 142:3

18. Brat R, Yousef N, Klifa R, Reynaud S, Shankar Aguilera S, De Luca D (2015) Lung ultrasonography score to evaluate oxygenation and surfactant need in neonates treated with continuous positive airway pressure. JAMA Pediatr 169(8):e151797

\section{Publisher's Note}

Springer Nature remains neutral with regard to jurisdictional claims in published maps and institutional affiliations.

\section{Submit your manuscript to a SpringerOpen ${ }^{\circ}$ journal and benefit from:}

- Convenient online submission

- Rigorous peer review

- Open access: articles freely available online

- High visibility within the field

- Retaining the copyright to your article

Submit your next manuscript at $\boldsymbol{\nabla}$ springeropen.com 